\title{
On the Principle of Optimality for Nonstationary Deterministic Dynamic Programming*
}

\author{
Takashi Kamihigashi ${ }^{\dagger}$
}

January 15, 2007

\begin{abstract}
This note studies a general nonstationary infinite-horizon optimization problem in discrete time. We allow the state space in each period to be an arbitrary set, and the return function in each period to be unbounded. We do not require discounting, and do not require the constraint correspondence in each period to be nonempty-valued. The objective function is defined as the limit superior or inferior of the finite sums of return functions. We show that the sequence of timeindexed value functions satisfies the Bellman equation if and only if its right-hand side is well defined, i.e., it does not involve $-\infty+\infty$.
\end{abstract}

Keywords: Bellman equation, dynamic programming, principle of optimality, value function

JEL Classification: C61, O41

\footnotetext{
*Some of the material of this note appeared in a preliminary version of my incomplete paper entitled "Nonlinear Duality for Dynamic Optimization," which now deals only with separate issues. I would like to thank Cuong Le Van and participants at various conferences and seminars for helpful comments.

${ }^{\dagger}$ RIEB, Kobe University, Rokkodai, Nada, Kobe 657-8501 JAPAN. Email: tkamihig@rieb.kobe-u.ac.jp. Tel/Fax: +81-78-803-7015.
} 


\section{Introduction}

The principle of optimality is a common tool in discrete-time optimization problems in economics. Bellman's (1957, p. 83) original version of the principle states that "an optimal policy has the property that whatever the initial state and initial decision are, the remaining decisions must constitute an optimal policy with regard to the state resulting from the first decision." An extended version of the principle says that the sequence of time-indexed value functions satisfies the Bellman equation. ${ }^{1}$ We call this version the extended principle of optimality in order to distinguish it from Bellman's original version. ${ }^{2}$ The extended principle follows from Bellman's principle if an optimal path is known to exist from every state in every period. However, if the existence of an optimal path is not guaranteed, the validity of the extended principle is less trivial.

Stokey and Lucas (1989, Chapter 4) dealt with this issue at the beginning of their analysis of the following problem:

$$
\begin{aligned}
\sup _{\left\{x_{t}\right\}_{t=1}^{\infty}} & \lim _{T \uparrow \infty} \sum_{t=0}^{T} \beta^{t} u\left(x_{t}, x_{t+1}\right) \\
\text { s.t. } & \forall t \in \mathbb{Z}_{+}, x_{t+1} \in \Gamma\left(x_{t}\right), \\
& x_{0} \in X \text { given, }
\end{aligned}
$$

where $\beta \geq 0, X$ is a set, and $\Gamma$ is a nonempty-valued correspondence from $X$ to itself. They also assumed that $u$ is finite valued and the limit in (1.1) exists in $\overline{\mathbb{R}}$ for any $x_{0} \in X$ and any path $\left\{x_{t}\right\}$ satisfying (1.2). Under these assumptions, they (Theorem 4.2) verified the extended principle of optimality, i.e., that the value function $v$ satisfies the Bellman equation:

$$
\forall x \in X, \quad v(x)=\sup _{y \in \Gamma(x)}\{u(x, y)+\beta v(y)\} .
$$

What is not clear from this well known result is the borderline between when the extended principle holds and when it fails. To our knowledge, the answer has not been documented in the literature even though the extended

\footnotetext{
${ }^{1}$ More precisely, it is often claimed in economics that value functions satisfy the Bellman equation by the "principle of optimality." This property of value functions is known as the "optimality equation" in the dynamic programming literature.

${ }^{2}$ See, e.g., Sniedovich $(1978,1992)$ for other versions of the principle of optimality.
} 
principle has been taken for granted or routinely verified for various problems that do not satisfy Stokey and Lucas's assumptions. The assumptions often violated are the stationarity of the problem (e.g., Hinderer, 1970; McKenzi, 1986; Michel, 1990), the existence of the limit in (1.1) for all feasible paths (McKenzi, 1986; Michel, 1990; Dana and Le Van, 1990), ${ }^{3}$ and the finiteness of $u$ (Alvarez and Stokey, 1998; Le Van and Morhain, 2002; Rincón-Zapatero and Rodríguez-Palmero, 2003). On the other hand, the validity of the "principle of optimality" has been questioned in various non-time-additive settings (e.g., Morin, 1982; Henig, 1985; Sniedovich, 1986; Tarvainen, 1995).

This note seeks to find the exact condition under which the extended principle holds, or fails, in the time additive case. For this purpose we make no assumption beyond the common framework of deterministic discrete-time optimization problems with time additive objective functions. In particular, we allow $X, u$, and $\Gamma$ to depend on time, allow $u_{t}$ to equal $-\infty$, do not require discounting, and do not require $\Gamma_{t}$ to be a nonempty-valued correspondence. We also replace lim in (1.1) by limsup or liminf, so that the objective function is always well defined.

We show two results. First, the sequence of the value functions always satisfies a slightly modified version of the Bellman equation. The required modification is to remove the states $y$ in $\Gamma_{t}(x)$ such that $u_{t}(x, y)=-\infty$. The proof of this result uses our preliminary lemma that the supremum over two variables can be split into two suprema. ${ }^{4}$ Given this result, our proof is similar to the classical argument (e.g., Bellman and Dreyfus, 1962, p. 15). Second, the value functions satisfy the Bellman equation if and only if its right-hand side is well defined or, more precisely, if it never happens that $u_{t}(x, y)=-\infty$ and $v_{t}(y)=\infty$ (where $v_{t}$ is the period $t$ value function). This is the precise condition under which the extended principle of optimality is valid. The purpose of this note is to clarify this point. Since we use lim sup or lim inf, instead of lim, to define the objective function, our results apply to models with the overtaking criterion (Gale, 1967) or weak maximality (Brock, 1970), provided that the return functions are appropriately normalized (e.g.,

\footnotetext{
${ }^{3}$ Strictly speaking, the paper by Dana and Le Van (1990) is not a good example here since they verified the existence of the limit in (1.1) for a normalized problem before establishing the extended principle; see Dana and Le Van $(1993,2006)$ for further results using this approach. Note however that their arguments can be simplified if the extended principle is known to be valid without the assumption that the limit exists.

${ }^{4}$ This is a simple extension of the "principle of conditional optimization" (Sniedovich, 1992).
} 
McKenzi, 1986; Michel, 1990).

The next section shows our preliminary lemma. Section 3 shows our main results.

\section{Splitting the Supremum over Two Variables}

This section shows a simple result that allows one to split the supremum over two variables into two suprema. Though the validity of this procedure is often taken for granted, it may not always be clear under what conditions it can be taken for granted. Our purpose here is to make it clear that the procedure is indeed valid without qualification. We do not claim originality.

We follow the convention that

$$
\sup \emptyset=-\infty .
$$

Hence $\sup A$ is always defined for any $A \subset \overline{\mathbb{R}}$. None of the sets in the following lemma is required to be nonempty.

Lemma 2.1. Let $X$ and $Y$ be sets, $\Pi \subset X \times Y$, and $f: \Pi \rightarrow \overline{\mathbb{R}}$. For $x \in X$ and $y \in Y$, define

$$
\begin{aligned}
& \Pi_{x}=\{y \in Y \mid(x, y) \in \Pi\}, \\
& \Pi_{y}=\{x \in X \mid(x, y) \in \Pi\} .
\end{aligned}
$$

Then

$$
\sup _{(x, y) \in \Pi} f(x, y)=\sup _{x \in X} \sup _{y \in \Pi_{x}} f(x, y)=\sup _{y \in Y} \sup _{x \in \Pi_{y}} f(x, y) .
$$

Proof. Let us show the first equality in (2.4). Note that

$$
\forall\left(x^{\prime}, y^{\prime}\right) \in \Pi, \quad f\left(x^{\prime}, y^{\prime}\right) \leq \sup _{y \in \Pi_{x^{\prime}}} f\left(x^{\prime}, y\right) \leq \sup _{x \in X} \sup _{y \in \Pi_{x}} f(x, y) .
$$

Thus

$$
\sup _{(x, y) \in \Pi} f(x, y) \leq \sup _{x \in X} \sup _{y \in \Pi_{x}} f(x, y) .
$$

To see the reverse inequality, note that

$$
\forall\left(x^{\prime}, y^{\prime}\right) \in \Pi, \quad \sup _{(x, y) \in \Pi} f(x, y) \geq f\left(x^{\prime}, y^{\prime}\right) .
$$


Hence

$$
\forall x^{\prime} \in X, \quad \sup _{(x, y) \in \Pi} f(x, y) \geq \sup _{y \in \Pi_{x^{\prime}}} f\left(x^{\prime}, y\right)
$$

Therefore

$$
\sup _{(x, y) \in \Pi} f(x, y) \geq \sup _{x \in X} \sup _{y \in \Pi_{x}} f(x, y) .
$$

Now the first equality in (2.4) follows from (2.6) and (2.9). The same argument shows the equality between the leftmost and the rightmost side of (2.4). Thus we obtain (2.4).

Since none of the sets above is required to be nonempty, the above result can be used whenever one has the supremum over two variables. Sniedovich (1992, p. 12) showed essentially the same result with max replacing sup. It is useful to note that the lemma enables one to interchange the order of "supremization" though this property is not used in this note.

\section{Main Results}

This section shows two results on the extended principle of optimality. Let us start with some notation.

For $t \in \mathbb{Z}_{+}$, let $X_{t}$ be a set, $\Gamma_{t}$ be a correspondence from $X_{t}$ to $X_{t+1}$, and

$$
D_{t}=\left\{\left(x_{t}, x_{t+1}\right) \in X_{t} \times X_{t+1} \mid x_{t+1} \in \Gamma_{t}\left(x_{t}\right)\right\} .
$$

For $t \in \mathbb{Z}_{+}$, let $u_{t}: D_{t} \rightarrow \mathbb{R} \cup\{-\infty\}$. The set $X_{t}$ is the state space in period $t, \Gamma_{t}$ is the constraint correspondence in period $t, u_{t}$ is the return function in period $t$, and $D_{t}$ is the domain of $u_{t}$.

For $t \in \mathbb{Z}_{+}$and $x_{t} \in X_{t}$, the set of paths feasible from $x_{t}$ is given by

$$
\Pi_{t}\left(x_{t}\right)=\left\{\left\{x_{i}\right\}_{i=t+1}^{\infty} \in X_{t+1} \times X_{t+2} \times \cdots \mid \forall i \geq t, x_{i+1} \in \Gamma_{i}\left(x_{i}\right)\right\} .
$$

Let $\mathrm{L}$ be liminf or limsup. (The definition of $\mathrm{L}$ is fixed from here on.) We consider the following problem:

$$
\begin{aligned}
\sup _{\left\{x_{t}\right\}_{t=1}^{\infty} \in \Pi_{0}\left(x_{0}\right)} & \underset{T \uparrow \infty}{\mathrm{L}} \sum_{t=0}^{T} u_{t}\left(x_{t}, x_{t+1}\right) \\
\text { s.t. } & \forall t \in \mathbb{Z}_{+}, x_{t+1} \in \Gamma_{t}\left(x_{t}\right), \\
& x_{0} \in X_{0} \text { given. }
\end{aligned}
$$


Since $u_{t}(x, y)<\infty$ for all $(x, y) \in D_{t}$, the objective function is always well defined.

For $t \in \mathbb{Z}_{+}$and $x_{t} \in X_{t}$, define the value function by

$$
v_{t}\left(x_{t}\right)=\sup _{\left\{x_{i}\right\}_{i=t+1}^{\infty} \in \Pi_{t}\left(x_{t}\right)} \underset{T \uparrow \infty}{\mathrm{L}} \sum_{i=t}^{T} u_{i}\left(x_{i}, x_{i+1}\right) .
$$

The associated Bellman equation is

$$
v_{t}\left(x_{t}\right)=\sup _{x_{t+1} \in \Gamma_{t}\left(x_{t}\right)}\left\{u_{t}\left(x_{t}, x_{t+1}\right)+v_{t+1}\left(x_{t}\right)\right\} .
$$

We wish to identify exactly when this equation holds, or fails. The following result shows that a slightly modified version of the equation always holds.

Theorem 3.1. For all $t \in \mathbb{Z}_{+}$and $x_{t} \in X_{t}$,

$$
v_{t}\left(x_{t}\right)=\sup _{x_{t+1} \in \hat{\Gamma}_{t}\left(x_{t}\right)}\left\{u_{t}\left(x_{t}, x_{t+1}\right)+v_{t+1}\left(x_{t+1}\right)\right\}
$$

where

$$
\hat{\Gamma}_{t}\left(x_{t}\right)=\left\{x_{t+1} \in \Gamma_{t}\left(x_{t}\right) \mid u_{t}\left(x_{t}, x_{t+1}\right)>-\infty\right\} .
$$

Proof. Let $t \in \mathbb{Z}_{+}$and $x_{t} \in X_{t}$. Define

$$
\hat{\Pi}_{t}\left(x_{t}\right)=\left\{\left\{x_{i}\right\}_{i=t+1}^{\infty} \in \Pi_{t}\left(x_{t}\right) \mid x_{t+1} \in \hat{\Gamma}\left(x_{t}\right)\right\} .
$$

Since $\mathrm{L}_{T \uparrow \infty} \sum_{i=t}^{T} u_{i}\left(x_{i}, x_{i+1}\right)=-\infty$ for $\left\{x_{i}\right\}_{i=t+1}^{\infty} \notin \hat{\Pi}_{t}\left(x_{t}\right)$, we have

$$
v_{t}\left(x_{t}\right)=\sup _{\left\{x_{i}\right\}_{i=t+1}^{\infty} \in \hat{\Pi}_{t}\left(x_{t}\right)} \underset{T \uparrow \infty}{\mathrm{L}} \sum_{i=t}^{T} u_{i}\left(x_{i}, x_{i+1}\right) .
$$

Let $\hat{\Gamma}=\hat{\Gamma}_{t}\left(x_{t}\right)$ and $\Pi_{x_{t+1}}=\Pi_{t+1}\left(x_{t+1}\right)$. Recalling (3.9), applying Lemma 2.1 
with $x=x_{t+1}$ and $y=\left\{x_{i}\right\}_{i=t+2}^{\infty},{ }^{5}$ we get

$$
\begin{aligned}
v_{t}\left(x_{t}\right) & =\sup _{\left\{x_{i}\right\}_{i=t+1}^{\infty} \in \hat{\Pi}_{t}\left(x_{t}\right)}\left\{u_{t}\left(x_{t}, x_{t+1}\right)+\underset{T \uparrow \infty}{\mathrm{L}} \sum_{i=t+1}^{T} u_{i}\left(x_{i}, x_{i+1}\right)\right\} \\
& =\sup _{x_{t+1} \in \hat{\Gamma}}\left\{\sup _{\left.x_{i}\right\}_{i=t+2}^{\infty} \in \Pi_{x_{t+1}}}\left\{u_{t}\left(x_{t}, x_{t+1}\right)+\underset{T \uparrow \infty}{\mathrm{L}} \sum_{i=t+1}^{T} u_{i}\left(x_{i}, x_{i+1}\right)\right\}\right. \\
& =\sup _{x_{t+1} \in \hat{\Gamma}}\left\{u_{t}\left(x_{t}, x_{t+1}\right)+\underset{\left\{x_{i}\right\}_{i=t+2}^{\infty} \in \Pi_{x_{t+1}}}{\operatorname{Lip\infty }} \sum_{i=t+1}^{T} u_{i}\left(x_{i}, x_{i+1}\right)\right\} \\
& =\sup _{x_{t+1} \in \hat{\Gamma}}\left\{u_{t}\left(x_{t}, x_{t+1}\right)+v_{t+1}\left(x_{t+1}\right)\right\} .
\end{aligned}
$$

Now (3.8) follows.

The following example illustrates why $\hat{\Gamma}_{t}\left(x_{t}\right)$ in (3.8) cannot be replaced by $\Gamma_{t}\left(x_{t}\right)$ in the above result. Let

$$
x_{0}=1, \quad \Gamma_{0}(1)=\{0,1\}, \quad \forall t \in \mathbb{N}, \Gamma_{t}\left(x_{t}\right)=\left\{x_{t}\right\} .
$$

Hence the only choice to be made is $x_{1} \in\{0,1\}$, and any feasible path $\left\{x_{t}\right\}_{t=1}^{\infty}$ is either the infinite sequence of 0 or that of 1 . Suppose

$$
\begin{array}{lll}
u_{0}(1,0)=0, & u_{0}(1,1)=-\infty, \\
\forall t \in \mathbb{N}, & u_{t}(0,0)=0, & u_{t}(1,1)=1 .
\end{array}
$$

Then for $\left\{x_{t}\right\}_{t=1}^{\infty} \in \Pi_{0}\left(x_{0}\right)$,

$$
\underset{T \uparrow \infty}{\mathrm{L}} \sum_{t=0}^{T} u_{t}\left(x_{t}, x_{t+1}\right)= \begin{cases}0 & \text { if } x_{1}=0, \\ -\infty & \text { if } x_{1}=1 .\end{cases}
$$

But by (3.18), $v_{1}(1)=\infty$. Thus the right-hand side of (3.8) would not be well defined if $\hat{\Gamma}_{t}\left(x_{t}\right)$ were replaced by $\Gamma_{t}\left(x_{t}\right)$.

A natural question is then whether the Bellman equation holds if its right-hand side does not involve $-\infty+\infty$. The answer is affirmative, as the following result shows.

\footnotetext{
${ }^{5}$ In addition, $\Pi=\hat{\Pi}_{t}\left(x_{t}\right), X=\hat{\Gamma}$, and $\Pi_{x}=\Pi_{x_{t+1}}$.
} 
Theorem 3.2. Let $t \in \mathbb{Z}_{+}$and $x_{t} \in X_{t}$. Then the Bellman equation (3.7) holds ${ }^{6}$ if and only if there exists no $x_{t+1} \in \Gamma_{t}\left(x_{t}\right)$ such that

$$
u_{t}\left(x_{t}, x_{t+1}\right)=-\infty, \quad v_{t+1}\left(x_{t+1}\right)=\infty .
$$

Proof. Let $t \in \mathbb{Z}_{+}$and $x_{t} \in X_{t}$. The necessity part is trivial since if there is $x_{t+1} \in \Gamma_{t}\left(x_{t}\right)$ satisfying (3.20), then the right-hand side of the Bellman equation is not well defined. To see the sufficiency part, suppose there is no $x_{t+1} \in \Gamma_{t}\left(x_{t}\right)$ satisfying (3.20). Then

$$
\forall x_{t+1} \in \Gamma_{t}\left(x_{t}\right) \backslash \hat{\Gamma}_{t}\left(x_{t}\right), \quad u_{t}\left(x_{t}, x_{t+1}\right)+v_{t+1}\left(x_{t+1}\right)=-\infty .
$$

Thus the Bellman equation (3.7) follows from (3.8).

This result shows that the extended principle of optimality holds if and only if the right-hand side of the Bellman equation is well defined. A simple sufficient condition for this is that $v_{t}\left(x_{t}\right)<\infty$ for all $t \in \mathbb{Z}_{+}$and $x_{t} \in X_{t}$. Since L may be limsup or liminf, Theorem 3.2 applies to models with the overtaking criterion (Gale, 1967) or weak maximality (Brock, 1970), provided that the return functions are appropriately normalized (e.g., McKenzi, 1986, p. 1287; Michel, 1990, p. 708). ${ }^{7}$

\section{References}

Alvarez, F., N. Stokey, 1998, Dynamic programming with homogenous functions, Journal of Economic Theory 82, 167-189.

Bellman, R., 1957, Dynamic Programming. Princeton University Press: Princeton.

Bellman, R.E., S.E. Dreyfus, 1962, Applied Dynamic Programming. Princeton University Press: Princeton.

Brock, W.A., 1970, On existence of weakly maximal programmes in a multisector economy, Review of Economic Studies 37, 275-280.

Dana, R.-A., C. Le Van, 1990, On the Bellman equation of the overtaking criterion, Journal of Optimization Theory and Applications 67, 587-600.

\footnotetext{
${ }^{6}$ We follow the convention that "an equation holds" means "both sides of it are well defined and are equal.

${ }^{7}$ In the literature it has been more common to assume $\mathrm{L}=\lim$ inf in order to preserve the concavity of value functions.
} 
Dana, R.-A., C. Le Van, 1993, On the Bellman equation of the overtaking criterion: addendum, Journal of Optimization Theory and Applications 78, 605-612.

Dana, R.-A., C. Le Van, 2006, Optimal growth without discounting, R.-A. Dana, C. Le Van, T. Mitra, K. Nishimura eds., Handbook of Optimal Growth 1: Discrete Time, pp. 1-17.

Gale, D., 1967, On optimal development in a multi-sector economy, Review of Economic Studies 34, 1-18.

Henig, M.I., 1985, The principle of optimality in dynamic programming with returns in partially ordered sets, Mathematics of Operations Research 10, 462-470.

Hinderer, K., 1970, Foundations of Non-stationary Dynamic Programming with Discrete Time Parameter. Springer-Verlag: Berlin.

Le Van, C., L. Morhaim, 2002, Optimal growth models with bounded or unbounded returns: a unifying approach, Journal of Economic Theory 105, $158-187$.

McKenzie, L.W., 1986, Optimal economic growth, turnpike theorems and comparative dynamics, K.J. Arrow, M.D. Intriligator, eds., Handbook of Mathematical Economics, Vol. 3, Amsterdam: North-Holland.

Michel, P., 1990, Some clarifications on the transversality condition, Econometrica 58, 705-723.

Morin, T.L., 1982, Monotonicity and the principle of optimality, Journal of Mathematical Analysis and Applications 86, 665-674.

Rincón-Zapatero, J.P., Rodríguez-Palmero, C., 2003, Existence and uniqueness of solutions to the Bellman equation in the unbounded case, Econometrica $71,1519-1555$.

Sniedovich, M., 1978, Dynamic programming and principles of optimality, Journal of Mathematical Analysis and Applications 65, 586-606.

Sniedovich, M., 1986, A new look at Bellman's principle of optimality, Journal of Optimization Theory and Applications 49, 161-176.

Sniedovich, M., 1992, Dynamic Programming. Marcel Dekker: New York.

Stokey, N., R.E. Lucas, Jr., 1989, Recursive Methods in Economic Dynamics. Cambridge, MA: Harvard University Press.

Tarvainen, K., 1995, An elimination condition to check the validity of the principle of optimality, Computers and Operations Research ? 23, 183192. 